\title{
A study of Babylonian planetary theory II. The planet Venus
}

\author{
Teije de Jong ${ }^{1}$
}

Received: 5 January 2019 / Published online: 29 March 2019

(c) The Author(s) 2019

\section{Abstract}

In this series of papers, I attempt to provide an answer to the question how the Babylonian scholars arrived at their mathematical theory of planetary motion. Paper I (de Jong in Arch Hist Exact Sci 73:1-37, 2019) was devoted to a study of system A theory of the outer planets. In this second paper, I will study system A theory of the planet Venus. All presently known ephemerides of Venus appear to have been written after $200 \mathrm{BC}$ so that the development of system A theory of Venus may have been a late development. On the other hand, there are several earlier texts in which the motion of Venus, going from one synodic phenomenon to the next one, is parametrized in quite some detail. At least six computational systems of Venus are known of which only two, systems $A_{0}$ and $A_{3}$, are genuine type-A systems. Both are based on the hypothesis that in exactly 1151 years Venus experiences 720 synodic phenomena of the same kind and that after this period of 1151 years Venus returns to exactly the same position in the sky. This period relation was probably derived from the observational fact that after 8 years Venus returns to a position in the zodiac that falls on average $2.5^{\circ}$ short of its previous position. The study of the Babylonian planetary theory of Venus presented here will be primarily based on the system $A_{3}$ theory of Venus because it is the most complete of the two systems. The parameters of the four step functions characterizing the system $\mathrm{A}_{3}$ model of the first and last appearances of Venus are known from previous studies of tablet BM 32599 (ACT 1050). Based on a database of synthetic observations of the first and last appearances (morning first, morning last, evening first and evening last) and the two stationary points (morning station and evening station) of Venus between $315 \mathrm{BC}$ and $50 \mathrm{BC}$, I first discuss the observational material from the point of view of a Babylonian astronomer. This involves deriving synodic time intervals and synodic arcs from the observed dates and longitudes of Venus. Both vary with the position of Venus in the zodiac. This variation shows up most purely in the synodic arcs and synodic time intervals of Venus at its stations. The variation pattern of the synodic

Communicated by Alexander Jones.

Teije de Jong

t.dejong@uva.nl

1 Astronomical Institute 'Anton Pannekoek', University of Amsterdam, Amsterdam, The Netherlands 
arcs and of the synodic time intervals at the first and last appearances of Venus differ considerably because they are strongly affected by atmospheric extinction and by the ecliptic latitude of Venus. A comparison of predictions of the first and last appearances of Venus computed according to system $\mathrm{A}_{3}$ with observations in the synthetic database shows that the models provide fairly accurate fits to the observations of evening last and morning first with typical standard deviations of about $2^{\circ}$ but rather poor fits to the observations of morning last and evening first with typical standard deviations of about $7^{\circ}$ and $4^{\circ}$. Quite surprisingly, it turns out that the morning first $\mathrm{A}_{3}$ model fits the observed longitudes of Venus at its stations almost perfectly, with standard deviations of about $1^{\circ}$. This suggests that observations of the stations of Venus must have been available to the Babylonian astronomer(s) who constructed the system $\mathrm{A}_{3}$ model of Venus and who computed the longitudes of Venus at its first and last appearances preserved in tablet BM 32599. This is quite puzzling because observations of the stations of Venus were definitely not part of the standard observing program of the Babylonian astronomers as we know it from the Astronomical Diaries. To resolve this problem, I propose that observations could have been made by some individual astronomer for his own use but that these observations never became part of the tradition of what was regularly observed and recorded in the Astronomical Diaries. It turns out that 25 years of observations of Venus (here illustrated for the years 203 BC to 178 BC) is sufficient for the construction of a system A model that perfectly fits the variation of the synodic arcs of Venus at its stations. By combining observed time intervals and known velocities of Venus, observations over this limited timespan can also be used to construct longitudes of Venus at its first and last appearances from previous known positions of Venus based on Normal Stars passages or from known positions at its stations. This is the only way in which positions of Venus at its first and last appearances can be determined because at its first and last appearances the sky is too bright for Normal Stars to be visible. I propose that the Babylonian system $\mathrm{A}_{3}$ models of Venus were based on longitudes constructed in this way. I finally suggest that the tablet, of which BM 32599 is the remainder, was filled line by line starting with a set of initial values in the bottom left-hand corner of the tablet and that the choice of these initial values was based on an observation of Venus at its evening station when it was exceptionally close to the Normal Star $\eta$ Piscium in February 179 BC. Based on this choice of initial conditions, the tablet covers the years $403 \mathrm{BC}$ to $170 \mathrm{BC}$ and it may have been composed around $170 \mathrm{BC}$. It was probably computed with the purpose of creating a raster of longitudes with mazes of about $2.5^{\circ}$ that could be used to predict future positions of Venus at its first or last appearance once a position at a previous first or last appearance was available.

\section{Introduction}

In this series of papers, I study the astronomical concepts, the inventions and the innovations underlying the development of Babylonian planetary theory. The central question to be answered is: "How did the Babylonian astronomers proceed from observation to theory?" Trying to provide an answer to this question is a real challenge because no texts are preserved in which the Babylonian scholars tell us how 
this was done. What we do have are observational texts, the so-called Astronomical Diaries and Excerpt Texts (Sachs and Hunger 1988, 1989, 1996; Hunger et al. 2001; henceforth referred to as the Diaries or ADRT I-III and V) and the finished theoretical products, the lunar and planetary ephemerides ${ }^{1}$ published in Astronomical Cuneiform Texts (Neugebauer 1955, henceforth ACT). The preserved Diaries provide a (strongly fragmented) continuous record of standardized naked-eye astronomical observations covering a period of about six centuries from about 650 to $50 \mathrm{BC}$, and the preserved ephemerides cover a period of about three centuries from about 310 to $50 \mathrm{BC}$.

I believe that the development of Babylonian planetary theory must have been a gradual stepwise process that may have taken more than a century. Since a zodiacal coordinate system is a prerequisite for a theoretical description of planetary motion and since we know that the $360^{\circ}$ Babylonian zodiac was introduced sometime during the fifth century $\mathrm{BC}$, this development probably took place during the fifth and fourth centuries $\mathrm{BC}$ preceding the appearance of the oldest preserved ephemeris (of the planet Mercury) for the years 309-289 BC (ACT No. 300). This timeframe is consistent with the discovery of an early system A-type scheme to compute longitudes of the last appearance of the planet Mercury (Aaboe et al. 1991, text M) that can be dated to around 400 BC. In a previous paper, I have suggested (de Jong 2017) that the oldest elements of Babylonian lunar theory may date from the late sixth/early fifth century $\mathrm{BC}$ so that the development of lunar system A theory probably preceded planetary theory. In paper I of this series (de Jong 2019), I have discussed the Babylonian system A theory of the outer planets Saturn, Jupiter and Mars. This paper is devoted to a study of the planet Venus, and in a third forthcoming paper I will treat the planet Mercury.

Our knowledge of the Babylonian theory of Venus is particularly fragmentary because of the small number and the bad state of preservation of ephemerides and procedure texts of this planet. On the other hand, the few available texts leave no doubt that a refined theory for Venus existed. For instance, in section 27 of the procedure text ACT No. 812 we read that the first appearance in the evening (EF) ${ }^{2}$ of Venus and the next stationary point (ES) are at an angular distance of 4,$19 ; 15^{\circ}$ if EF occurs in Virgo and that the last visibility in the evening (EL) then occurs after a retrograde motion of $6 ; 15^{\circ}$ (ACT, 300). Since the publication of ACT, a few additional procedure texts for Venus have been discovered and one text, ACT 1050, ${ }^{3}$ that had provisionally been assigned to Mercury, turned out to contain predictions of the longitudes of Venus at its first and last appearances (Hamilton and Aaboe 1998). The present state

\footnotetext{
${ }^{1}$ Following Neugebauer (ACT, 1), I will use the term ephemerides for texts which contain "lists of positions of the sun, the moon and the planets computed for regular time intervals".

2 In this paper, I will use the acronyms MF (first appearance in the morning), MS (morning station) and ML (last appearance in the morning) and EF (first appearance in the evening), ES (evening station) and EL (last appearance in the evening) proposed by Ossendrijver (2012, 56-58). In the older literature, one often encounters the Greek capital letters $\Gamma, \Phi, \Sigma, \Xi, \Psi$ and $\Omega$ that were introduced by Neugebauer (ACT, 280; see also HAMA, 386) to represent the "characteristic phenomena" of the inner planets.

3 This text, also known as BM 32599, is part of a collection of tablets that was assembled in Iraq by George Smith and that arrived after his death (Aleppo, August 1876) at the British Museum. The tablet is mentioned by Strassmaier in the historical introduction of his pioneering publication "Zur Entzifferung der astronomischen Tafeln der Chaldäer" and is probably the first cuneiform text to have been recognized as containing astronomical tabular material (see de Jong 2016, 296-297).
} 
of our knowledge of the Babylonian theory of Venus has recently been summarized by Ossendrijver (2012, 75-83).

At least six computational systems of Venus are known of which only two, systems $\mathrm{A}_{0}$ and $\mathrm{A}_{3}$, are genuine type-A systems. In a system A planetary theory, the variation of the synodic arc with position in the zodiac is approximated by a step function such that the zodiac is divided in a number of zones with different constant values of the synodic arc. Systems $\mathrm{A}_{1}, \mathrm{~A}_{2}, \mathrm{C}_{3}$ and $\mathrm{C}_{4}$ are pseudo-type-A systems because the algorithm for the synodic arc does not contain transition rules between different zones (Ossendrijver 2012, 75).

System $\mathrm{A}_{0}$ is represented by only one ephemeris (ACT 400) which contains dates and longitudes of the first appearance of Venus as evening star (EF) for twenty-four consecutive years. It was written by the well-known astronomer Anu-aba-utêr from Uruk which implies a date within the range SE 110-124 (202-188 BC). System $\mathrm{A}_{3}$ is represented by ACT 1050 which in its original state contained a synodic table for EF, EL, MF and ML followed by several brief computational procedures (Hamilton and Aaboe 1998; Britton 2001). Since the synodic table contains only zodiacal positions, nothing is known about the algorithm for the dates. Britton (2001) dates this text to around 186 BC (SE 126). The study of the Babylonian planetary theory of Venus presented here will be primarily based on the system $A_{3}$ theory of Venus because it is the most complete of the two systems.

All presently known ephemerides of Venus appear to have been written after $200 \mathrm{BC}$ so that the development of system A theory of Venus may have been a late development. On the other hand, there are several earlier texts in which the motion of Venus, going from one synodic phenomenon to the next one, is computed in great detail. In BM 36301, dated to around $360 \mathrm{BC}$, we find longitudes and dates of Venus through one complete synodic cycle. The synodic data in this text turn out to be computed with a fairly primitive scheme to model the variable motion of Venus from MS to ES and with a completely unrealistic scheme from ES to MS where Venus moves prograde rather than retrograde (Neugebauer and Sachs 1967; Britton and Walker 1991). The somewhat later computational scheme used in the text BM 33552, dating from around $320 \mathrm{BC}$, is already much more realistic by dividing the synodic cycle in 8 zones with different constant velocities and proper treatment of the retrograde motion of Venus from ES to MS. The most refined computational scheme for the variable motion of Venus is found in the late text BM 37151, dating from around 120 BC. In this badly preserved text, the synodic cycle is divided in 11 zones with different constant velocities, while for each of the five successive synodic cycles during one 8-year period different values for the synodic time intervals are used (Aaboe and Huber 1977; Britton and Walker 1991).

Both system A theories of Venus are based on the hypothesis that in exactly 1151 years Venus experiences 720 synodic phenomena of the same kind and that after this period of 1151 years Venus returns to exactly the same position in the sky. This implies that in 1151 years Venus, being an inner planet, transgresses the sky 1151 times while it orbits the Sun $1151+720=1871$ times. ${ }^{4}$ Because of the great length

\footnotetext{
4 These numbers imply a synodic period for Venus of $1151 / 720$ years $=583.88$ days and an orbital period of $1151 / 1871$ years $=224.69$ days, quite accurate compared to modern values of 583.92 and 224.70 days, respectively.
} 
of this period, there can be no doubt that these parameters are not directly observed but must be the result of computation by somehow combining period relations with considerable shorter periods.

Indeed, several much shorter periods of Venus are attested in the Babylonian astronomical literature (Britton 2002; Brack-Bernsen and Hunger 2005/2006). All these periods are multiples of the well-known 8-year Venus cycle that may already have been known in old Babylonian times (see Reiner and Pingree 1975). In 8 years, Venus reappears and disappears five times as morning star and as evening star and orbits the Sun thirteen times. It reappears or disappears very close to the position in the sky at which it appeared or disappeared 8 years before. The magnitude of this shift in position after 8 years is about $2.5^{\circ}$ backwards (to the West).

The derivation of the 1151-year ephemeris period of Venus may be understood if we assume that the Babylonian scholars were aware of the fact that the position of Venus was shifted backwards by $2.5^{\circ}$ in the ecliptic at reappearance after 8 years. This implies that after $360^{\circ} / 2.5^{\circ}=1448$-year cycles Venus would return to exactly the same position in the zodiac at its reappearance, having completed $144 \times 8-1=1151$ rounds of the sky in 1151 years and having reappeared as morning star $144 \times 5=720$ times during that period. The question how they arrived at the 1151-year ephemeris period then reduces to how they established the $2.5^{\circ}$ backward shift in the position of Venus in one 8-year cycle.

Since after 720 synodic periods Venus has completed 1151 sidereal rotations, we may write $1151 \times 360^{\circ}=720 \times \overline{\Delta \lambda}$ so that the mean synodic arc of Venus, i.e. the average increase in longitude of Venus between two successive first or last appearances, equals $\overline{\Delta \lambda}=575 ; 30^{\circ}$, equivalent to one full passage through the zodiac of $360^{\circ}$ plus in addition a synodic arc of $215 ; 30^{\circ}$.

The synodic time interval is defined as the time measured in lunar months and days (or tithis) between two successive first or last appearances of Venus. The period relation for Venus, where 720 synodic time intervals occur in 1151 Babylonian years of 12;22,08 months of 30 tithis each, results in the relation $720 \times(360+\overline{\Delta t})=1151 \times$ $12 ; 22,08 \times 30$, where $\overline{\Delta t}$ is the mean synodic time step, i.e. the excess time in tithis of the synodic period over the length of the Babylonian year of 12 lunar months (of 30 tithis each). We then find $\overline{\Delta t}=233 ; 11,29$ tithis.

A key concept in Babylonian planetary theory is that synodic time intervals can be computed from synodic arcs, and vice versa, by using the difference $c=\overline{\Delta t}-$ $\overline{\Delta \lambda}$, derived from the period relation. For Venus, we find $c=233 ; 11,29-215 ; 30=$ $17 ; 41,29$, which is usually rounded off to $17 ; 40$.

In principle, we now could compute a zero-order average Babylonian ephemeris for the first or last appearances of Venus, starting with an observed initial position $\lambda_{0}$ at $t_{0}$ by progressing the longitude of Venus in steps of $\overline{\Delta \lambda}=360^{\circ}+215 ; 30^{\circ}$ and the time in time steps of $\overline{\Delta t}=12$ lunar months $+233 ; 11,29$ tithis, such that after $n$ steps we have $\lambda_{\mathrm{n}}=\lambda_{0}+n \cdot \overline{\Delta \lambda}$, and $\mathrm{t}_{\mathrm{n}}=\mathrm{t}_{0}+12 n$ lunar months $+n \cdot \overline{\Delta t}$. It is interesting to note that this simple computational algorithm is applied in two preserved Venus ephemerides. In ACT No. 400 (system $\mathrm{A}_{0}$ ), it is used to predict the positions and dates for the first appearance of Venus in the evening (EF), and in ACT 1050 (system $A_{3}$ ) it is used to compute the positions of Venus at its last appearance in the evening (EL). This algorithm produces a rather poor model for EF of Venus with errors in position 
up to about one zodiacal sign, but, as we shall demonstrate below, it provides a quite accurate approximation to the position of Venus at EL.

An important limitation in all theoretical work on Babylonian planetary theory is that at first and last appearances of a planet its position (relative to one of the Normal Stars) is in general impossible to establish by direct observation because when it reappears at dawn or disappears at dusk close to the horizon, no nearby stars are visible. This is in particular true for the planet Venus because it is brighter than any star in the sky. In paper I, it was shown that for the outer planets this difficulty was overcome by the Babylonian astronomers by using observations of two other synodic phases of the planets: the stationary points in their orbits. Therefore, one might expect that a similar approach was used in the development of the planetary theory of Venus. At its stationary points, Venus is at a distance of about $30^{\circ}$ from the Sun so that there is indeed about one hour of dark night after it sets or before it rises in which the position of Venus relative to nearby stars in the ecliptic can be observed (the Babylonian Normal Stars). This is a straightforward observation that one would expect to have been part of the standard observational program in the Astronomical Diaries. While the Diaries contain numerous observations of the stationary points of the outer planets, strangely enough there is only one record of such an observation ${ }^{5}$ for Venus. This is all the more puzzling in view of the fact that the Venus ephemerides ACT 410 and 420, computed according to the pseudo-systems $\mathrm{A}_{1}$ and $\mathrm{A}_{2}$, list dates and positions of both the morning and the evening stationary points of Venus (MS and ES) for several tens of years during the last two centuries BC.

\section{The Babylonian observational database}

To illustrate the difficulties that confronted the Babylonian scholars in their attempt to model the motion of the planet Venus, I show in Fig. 1 a typical example of its apparent motion during 23 months, from 5 April 148 BC to 6 March 146 BC when Venus makes two full passages through the ecliptic. Within this time frame, Venus completes one synodic period, from its first appearance in the evening on 11 June $148 \mathrm{BC}$ to its next first appearance in the evening on 21 January 146 BC, in 589 days. Notice that Venus moves from right to left in the figure and from West to East in the sky. Venus is a so-called inner planet because its orbital radius is smaller than that of the Earth so that it revolves around the Sun within the Earth orbit. This implies that the angular distance of Venus to the Sun in the sky is always less than about $45^{\circ}$ so that Venus is visible as morning star before sunrise in the Eastern morning sky or as evening star after sunset in the Western evening sky. Another consequence of this orbital configuration is that Venus experiences first and last appearances both as morning star and as evening star. In Fig. 1, Venus moves from its first appearance as evening star (EF) in 243 days to its evening station (ES) where it reverses its motion from prograde to retrograde, and then in 17 days moves backward to its last appearance in the evening (EL). After a short

\footnotetext{
5 This observation is recorded in Astronomical Diary No. -137 (ADRT III, 185) on day 29 of month XII ${ }^{2}$ in SE 175 (14/15 April 136 BC). There we read: “... when Venus became stationary to the east, it became stationary 2 fingers in front of $\beta$ Tauri, 1 finger low to the south", where 1 finger corresponds to about 5 arc minutes.
} 

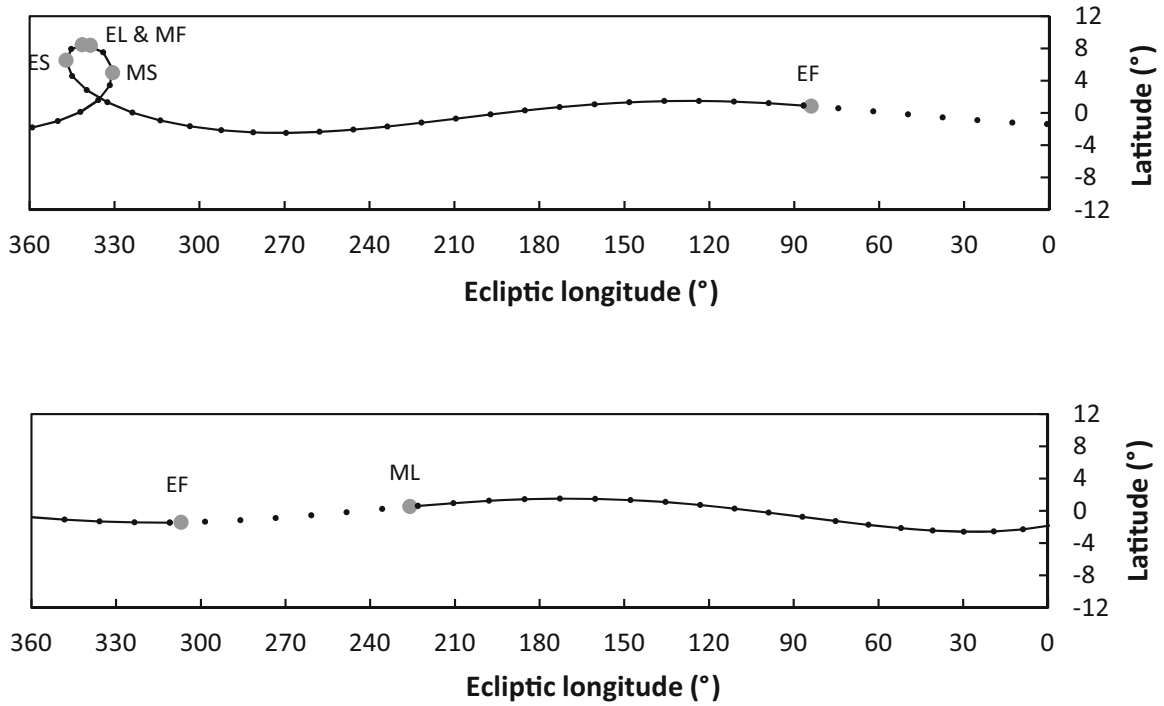

Fig. 1 The apparent motion of the planet Venus from 5 April 148 BC to 11 May 147 BC (upper panel) and from 12 May $147 \mathrm{BC}$ to 6 March $146 \mathrm{BC}$ (lower panel). Positions of Venus at 10 day time intervals are represented by small black dots. Synodic phenomena of Venus are represented by large grey dots. Venus completes one synodic period, from its first appearance in the evening on 11 June 148 BC to its next first appearance in the evening on 21 January 146 BC, in 589 days. It moves from right to left in the figure and from West to East in the sky

period of invisibility of 4 days, Venus reappears as morning star (MF), still moving backwards in 20 days to its morning station (MS), where it resumes its prograde motion, and from there in 240 days moves to its last appearance in the morning (ML). After having been invisible for 64 days, it reappears at its next first appearance in the evening (EF), thereby completing one synodic period. ${ }^{6}$ Notice the large ecliptic latitude of Venus in the region of the sky where it reverses its motion. Typical angular distances traversed by Venus going from one synodic phenomenon to the next can be easily read off in Fig. 1.

The synodic phenomena, sometimes also referred to as synodic phases, of the planets were extensively observed by the Babylonian astronomers, and the dates on which the planets first appeared, reached their stationary points and finally disappeared were systematically recorded in the Astronomical Diaries. It is important to realize that the mean synodic period of a planet, the average time interval after which it returns to its next similar synodic phenomenon, is the same for all synodic phenomena. This is due to the fact that each synodic phenomenon of a planet is characterized by a specific value of its solar elongation, the angular distance of the planet to the Sun as seen from the Earth. ${ }^{7}$

\footnotetext{
6 To better understand the way in which the orbital configuration of an inner planet determines the observational behaviour of Venus, its first and last appearances as evening and morning star and its periods of invisibility, the reader may be referred to Fig. 1 of Reiner and Pingree (1975).

7 In present-day astronomy, the synodic period is defined as the time it takes for a planet to move from one conjunction of the Sun to the next one. At conjunction, the solar elongation of a planet is by definition
} 
We know that the Babylonian scholars compiled long lists of observations of synodic phenomena of the planets (first and last appearances and stations) covering many tens of years (ADRT V). These lists were extracted from the observational data routinely recorded in the Astronomical Diaries. For first and last appearances, they listed dates and sometime zodiacal signs, and for the stations they listed dates and angular distances to nearby Normal Stars. This is related to the fact that positions of planets with respect to nearby stars can only be determined at night when the sky is dark and not when planets experience their first or last appearance because during twilight the sky is too bright for stars to be visible. Approximate positions of planets in zodiacal signs can be estimated by extrapolating from earlier nights when nearby stars were still visible.

From the observations of positions and dates of planets at successive synodic phases, synodic arcs and synodic time intervals can be computed which must have served as the basic data from which the Babylonian scholars constructed their planetary models. To be able to study this observational material and to attempt to understand the way in which it may have been used for the construction of the system $A_{3}$ model of the planet Venus, I have created a synthetic observational database by computing the dates and zodiacal longitudes of all first and last appearances and of all stationary points of Venus in Babylon during the Seleucid Era (here loosely defined as lasting from 315 to $50 \mathrm{BC}){ }^{8}$ These computations are based on modern astronomical theory and on the physical visibility criterion that I used in previous studies of first and last appearances of stars and planets (see de Jong 2012 and references therein), adopting a nominal atmosphere in Babylon characterized by a visual extinction of 0.27 magnitudes per air mass. ${ }^{9}$

The synthetic database consists of lists of dates of the first and last appearances of Venus and of its stations in the Babylonian lunar calendar and of the zodiacal longitudes of Venus on those dates in the Babylonian fixed sidereal zodiac similar to what we find in the Babylonian Excerpt Texts with the one important difference that the longitudes of Venus in the synthetic database are given to a fraction of a degree, while the Excerpt Texts usually only give the zodiacal sign or an association with one of the Normal Stars. From the synthetic data, we may construct the synodic time interval and the synodic arc (the longitude interval) for Venus from one synodic phenomenon to the next similar one.

To be able to construct an ephemeris for a synodic phase of some planet, the Babylonian scholars needed the length of the synodic arc that had to be added to the longitude of the planet at that synodic phase to obtain the longitude at the next similar synodic phase. These synodic arcs vary with position of a planet in the zodiac

Footnote 7 continued

equal to zero. Each of the synodic phenomena of a planet is characterized by a specific value of the solar elongation. For Venus, we find solar elongations of $-8^{\circ}$ at $\mathrm{MF},-29^{\circ}$ at $\mathrm{MS},-10^{\circ}$ at $\mathrm{ML}, 9^{\circ}$ at $\mathrm{EF}, 29^{\circ}$ at ES and $12^{\circ}$ at EL.

8 Ecliptic longitudes of Venus in the database are converted to longitudes in the Babylonian fixed zodiac by using the relation (see Huber 1958) $\lambda_{\text {bab }}=\lambda_{\text {ecl }}+4^{\circ} 28^{\prime}-(T+100) / 71.6$, where $T$ is the Julian astronomical year (e.g. $484 \mathrm{BC}=-483$ ).

9 This atmospheric extinction corresponds to an arcus visionis of about $7^{\circ}$ for all first and last appearances of Venus. In my earlier study of Babylonian observations of Venus (de Jong 2012), I found that the arcus visionis differs for different synodic phases, varying from $6.1^{\circ}$ to $8.6^{\circ}$. Since we are here mainly interested in intervals of position and time, the fact that the database is composed of data which are calculated for one and the same value of the arcus visionis does not affect the results of the analysis. 

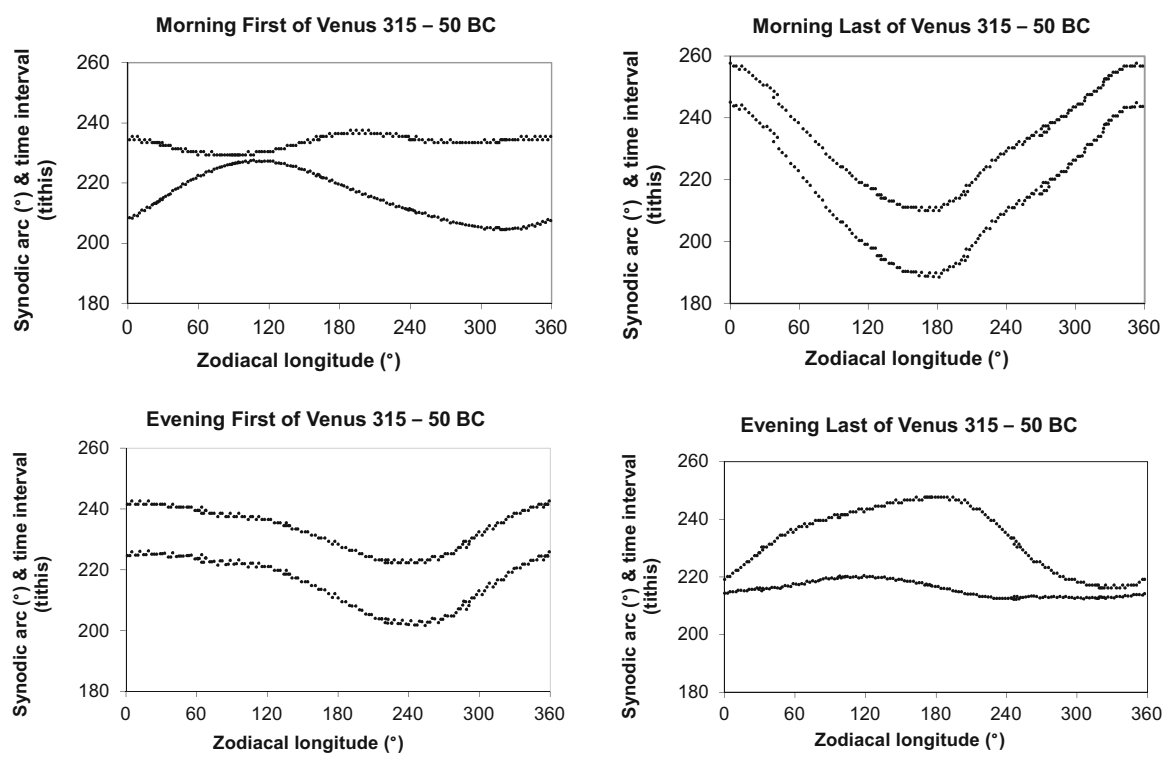

Fig. 2 Synodic arcs (lower curves) and synodic time intervals (upper curves) derived from synthetic observations of Venus during the Seleucid Era (315-50 BC) as a function of the Babylonian zodiacal longitude of Venus (black dots). Notice the large differences in the variation of the synodic arcs and the synodic time intervals between the four different synodic phases of Venus

because the angular velocity of a planet varies with position in its orbit. ${ }^{10}$ They also needed synodic time intervals to compute successive dates of that particular synodic phenomena. These synodic time intervals also vary with position of the planet in the sky. As discussed above, the Babylonian scholars computed synodic time intervals (in tithis) by adding a constant number $c$ to the synodic arc (in degrees). There we showed that for Venus $c=17 ; 40$.

In Fig. 2, I have plotted, for all four first and last appearances of Venus, the synodic arcs and the synodic time intervals that must be added to the longitude of Venus and to the date to obtain the longitude and the date of Venus at the next similar first or last appearance. The data in Fig. 2 are computed from the synthetic observational database. They are graphical representations of data that could have been computed by the Babylonian scholars from the observations of the first and last appearances of Venus collected in Excerpt Texts (ADRT V). In fact, these are the data that are required for the construction of a system A model of Venus. It is important to realize that of the observational data in Fig. 2, synodic time intervals can be directly computed from observed dates, while zodiacal longitudes of Venus and synodic arcs must be determined in some other way because positions of Venus with respect to Normal Stars cannot be determined at first or last appearances of Venus.

In paper I, we have seen that for the outer planets this difficulty was overcome by using observations of the planets at their stationary points, where longitudes can be

\footnotetext{
10 We know now that this is due to the eccentricity of the planetary orbit but for the Babylonians this was just an empirical fact.
} 

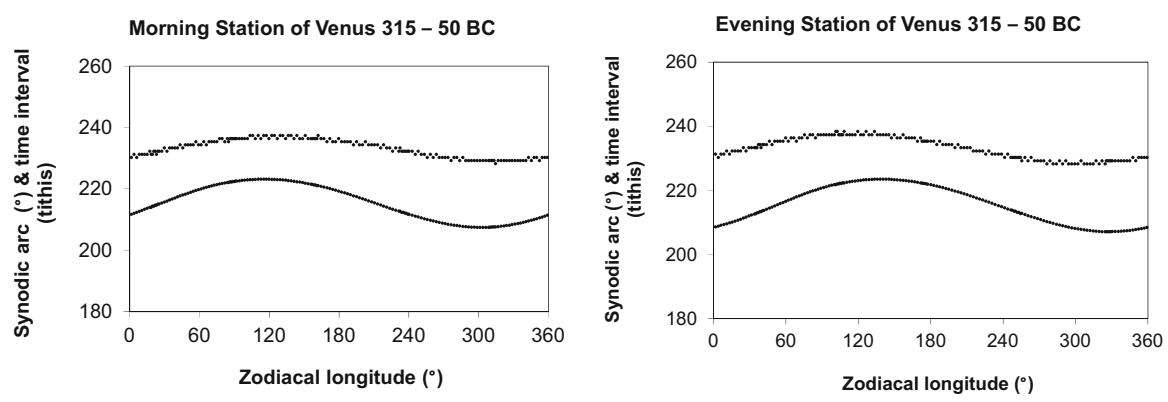

Fig. 3 Same as Fig. 2 but now for the morning and evening stations of Venus. Notice the similarity in the variation of the synodic arcs and the synodic time intervals between the two stations of Venus

Table 1 Average observed synodic intervals and Babylonian parameters for Venus

\begin{tabular}{lccc}
\hline \multicolumn{1}{c}{ Observation } & Synodic time step $\Delta \mathrm{t}$ (tithis) & Synodic $\operatorname{arc} \Delta \lambda\left(^{\circ}\right)$ & $\Delta \mathrm{t}-\Delta \lambda=\mathrm{c}$ \\
\hline Morning First & $233.2 \pm 2.3$ & $215.5 \pm 7.9$ & $17.7 \pm 9.5$ \\
Morning Station & $233.2 \pm 2.8$ & $215.6 \pm 5.6$ & $17.7 \pm 2.9$ \\
Morning Last & $233.1 \pm 15.6$ & $215.4 \pm 18.2$ & $17.7 \pm 2.7$ \\
Evening First & $233.2 \pm 6.8$ & $215.6 \pm 8.2$ & $17.7 \pm 1.8$ \\
Evening Station & $233.2 \pm 3.3$ & $215.6 \pm 5.8$ & $17.7 \pm 3.1$ \\
Evening Last & $233.1 \pm 11.0$ & $215.5 \pm 2.7$ & $17.7 \pm 9.4$ \\
Babylonian & $233 ; 11,29$ & $215 ; 30$ & $17 ; 40$ \\
& in excess of 12 months & in excess of $360^{\circ}$ & \\
\hline
\end{tabular}

(i)

(ii)

(iii)

(iv)

determined from the measurement of distances to nearby Normal Stars. However, as pointed out earlier, the observation of Venus near its stations does not seem to have been part of the standard Babylonian observing practice as recorded in the Astronomical Diaries. Nevertheless, since there are strong indications that the Babylonian scholars must have used observations of Venus near its stations for the construction of system $\mathrm{A}_{3}$, I show in Fig. 3 the same data as in Fig. 2, but now for the stations of Venus. Because there is about one hour of dark time to observe Venus at its morning station before sunrise and at its evening station after sunset, the data displayed in Fig. 3 can indeed be directly derived from observed longitudes and dates of Venus at its stations.

As expected from the relation $\overline{\Delta t}=\overline{\Delta \lambda}+17 ; 40$, we see that in Figs. 2 and 3 the synodic time intervals are larger than the synodic arcs, but not by $17 ; 40$ everywhere. However, as shown in Table 1, this relation still holds on average over all longitudes. It provides a good approximation for $\mathrm{EF}$ and $\mathrm{ML}$ and the stations but a rather poor approximation for MF and EL, where errors of up to 15 days may occur if synodic time intervals are computed from synodic arcs.

It is quite clear from Figs. 2 and 3 that the variable orbital motion of Venus is reflected in the variation of the synodic arcs, with amplitudes varying from $\pm 4^{\circ}$ at EL to $\pm 28^{\circ}$ for ML. This variation can be most purely observed in Fig. 3 where the variation of the synodic arcs of Venus is nearly identical for both stations with 
an amplitude of $\pm 8^{\circ} .{ }^{11}$ For the other synodic phases of Venus, EL and MF near inner conjunction and ML and EF near outer conjunction of Venus with the Sun, the variation pattern of the synodic arcs is strongly affected by a combination of ecliptic latitude and atmospheric extinction which plays an important role near the horizon. At inner conjunction of Venus with the Sun, when it is nearest to the Earth, its ecliptic latitude may become quite large with values up to $\pm 8^{\circ}$, while at outer conjunction the latitude of Venus varies within $\pm 1^{\circ}$. On the other hand, at inner conjunction Venus moves with a velocity of about $-1.5^{\circ}$ per day so that it may overcome the effect of the extinction near the horizon in a few days, while at outer conjunction where the relative velocity of Venus equals about $0.25^{\circ}$ per day, Venus has to struggle with the extinction for many days to finally become visible in the West after sunset (EF) or to eventually disappear in the East before sunrise (ML). I have verified by computing the synodic phenomena of Venus without ecliptic latitude in a transparent atmosphere that all curves in Fig. 2 reduce to those in Fig. 3 so that indeed the effects of ecliptic latitude and atmospheric extinction are responsible for the differences in the variation of the synodic arcs and the time intervals for the first and last appearances of Venus. There is much more to say about this topic, but a more detailed discussion of the astronomical and physical aspects of these phenomena is outside the scope of this paper. Moreover, the Babylonian scholars, largely unaware of these subtleties, were mainly concerned with modelling the empirical facts.

\section{The Babylonian system $A_{3}$ model of Venus}

As discussed above, the most complete model of Venus is system $\mathrm{A}_{3}$, first identified and analysed by Hamilton and Aaboe (1998) and later studied in more detail by Britton (2001). The parameters defining system $\mathrm{A}_{3}$ were extracted by Hamilton and Aaboe (1998) and Britton (2001) from BM 32599 (ACT 1050), a rather well-preserved tablet containing longitudes of first and last appearances of Venus. BM 32599 is the remaining right part of a tablet that originally contained twenty columns with twenty-nine entries, each line listing longitudes of Venus at successive first and last appearances. Twenty successive first and last appearances correspond to 8 years, the well-known period of Venus, so that the longitudes of Venus decrease on average by about $2.5^{\circ}$ going down from line to line in each column of the tablet. BM 32599 has sixteen lines written on the obverse and thirteen lines on the reverse side. The first eleven columns of the original tablet are lost, while the twelfth, which is only partly preserved on BM 32599, contains the longitudes of the third EF in the 8-year cycle. BM 32599 only lists longitudes, no dates. ${ }^{12}$ Contrary to most other Venus ephemerides, the stations are not included.

The parameters defining model $\mathrm{A}_{3}$ are collected in Table 2, where the parameters derived from the period relation are shown in columns (i)-(vi), and those characterizing

\footnotetext{
11 Close inspection of the data displayed in the two panels of Fig. 3 shows that the curves are shifted by about $25^{\circ}$ towards larger longitudes going from morning station to evening station. This shift is due to the elliptical shapes of the orbits of Venus and the Earth which affects the position of Venus in the sky where the synodic arc reaches its maximum or minimum value.

12 Ossendrijver (2012) refers to this type of texts, only containing longitudes of the synodic phenomena of the planets and no dates, as template tables.
} 
Table 2 Babylonian system $\mathrm{A}_{3}$ parameters for Venus

\begin{tabular}{|c|c|c|c|c|c|c|c|c|c|c|c|c|}
\hline $\begin{array}{l}\text { Period } \\
\text { (years) }\end{array}$ & $\begin{array}{l}\text { Synodic } \\
\text { periods }\end{array}$ & $\begin{array}{c}\text { Sidereal } \\
\text { rotations }\end{array}$ & $\begin{array}{c}\text { mean } \Delta \mathrm{t} \\
\text { (tithi's) }\end{array}$ & $\begin{array}{c}\text { mean } \Delta \lambda \\
\left({ }^{\circ}\right)\end{array}$ & $\mathrm{c}$ & Obs & $\mathrm{i}$ & $\begin{array}{l}\alpha(\mathrm{i}) \\
\left(^{\circ}\right)\end{array}$ & $\begin{array}{c}\lambda_{\text {init }}(\mathrm{i}) \\
\left({ }^{\circ}\right)\end{array}$ & $\begin{array}{c}\text { w(i) } \\
\left({ }^{\circ}\right)\end{array}$ & $\begin{array}{c}\mathrm{w}^{\prime}(\mathrm{i}) \\
\left(^{\circ}\right)\end{array}$ & w(i+1)/w(i) \\
\hline \multirow[t]{13}{*}{1151} & 720 & 1871 & $\begin{array}{c}12 \text { months } \\
233 ; 11,29\end{array}$ & $\begin{array}{l}360^{\circ}+ \\
215 ; 30\end{array}$ & $17 ; 41,29$ & $\begin{array}{l}\text { EL } \\
\text { MF }\end{array}$ & & $360 ; 00$ & $0 ; 00$ & $215 ; 30$ & $2 ; 30$ & \\
\hline & & & & & & & 1 & $84 ; 00$ & $18 ; 00$ & $210 ; 08$ & $2 ; 20$ & $15 / 14$ \\
\hline & & & & & & & 2 & $84 ; 00$ & $102 ; 00$ & $215 ; 30$ & $2 ; 30$ & $16 / 15$ \\
\hline & & & & & & & 3 & $96 ; 00$ & $186 ; 00$ & $229 ; 52$ & $2 ; 40$ & $15 / 16$ \\
\hline & & & & & & & 4 & $96 ; 00$ & $282 ; 00$ & $215 ; 30$ & $2 ; 30$ & $14 / 15$ \\
\hline & & & & & & ML & & & & & & \\
\hline & & & & & & & 1 & $174 ; 00$ & $102 ; 00$ & $215 ; 30$ & $2 ; 30$ & $8 / 9$ \\
\hline & & & & & & & 2 & $82 ; 40$ & $276 ; 00$ & $191 ; 33,20$ & $2 ; 13,20$ & $10 / 8$ \\
\hline & & & & & & & 3 & $103 ; 20$ & $358 ; 40$ & $239 ; 26,40$ & $2 ; 46,40$ & $9 / 10$ \\
\hline & & & & & & EF & & & & & & \\
\hline & & & & & & & 1 & $103 ; 20$ & $102 ; 00$ & $239 ; 26,40$ & $2 ; 46,40$ & $8 / 10$ \\
\hline & & & & & & & 2 & $82 ; 40$ & $205 ; 20$ & $191 ; 33,20$ & $2 ; 13,20$ & $9 / 8$ \\
\hline & Baby & lonian ado & oted value & & $17 ; 40$ & & 3 & $174 ; 00$ & $288 ; 00$ & $215 ; 30$ & $2 ; 30$ & $10 / 9$ \\
\hline (i) & (ii) & (iii) & (iv) & (v) & (vi) & (vii) & & (viii) & (ix) & $(\mathrm{x})$ & (xi) & (xii) \\
\hline
\end{tabular}

the step functions of the different synodic phases are given in columns (vii)-(xi). An important aspect of these models is that all of them are based on the same period relation but with a different set of parameters for each synodic phenomenon. Notice that the synodic arc in the model for EL is constant, equal to the average synodic arc (see column (v) of Table 2), that the synodic arc for MF is modelled by a fourfold step function and for ML and EF by threefold step functions. Also notice that the models for ML and EF are characterized by very similar parameters but distributed differently in the zodiac.

Graphical representations of the "observed" synodic arcs and of the system $\mathrm{A}_{3}$ models of the four synodic phenomena are shown in Fig. 4. Dots represent the synthetic observations taken from Fig. 2, and the thin lines represent the model $\mathrm{A}_{3}$ step functions. The full lines represent the real model values, which are smoothed versions of the defining step functions. This smoothing is due to the interpolation algorithm which must be applied when the amplitude $w(\mathrm{i})$ of the step function (column (x) of Table 2) exceeds the length $\alpha$ (i) of the zone (column (viii) of Table 2) so that more than one zonal boundary is transgressed in the computation of the synodic arc (Ossendrijver 2012, 527).

The data displayed in Fig. 4 show that the Babylonian system $\mathrm{A}_{3}$ model for the synodic arcs of Venus provides a reasonable first approximation to astronomical reality. The model values for MF and EL never differ more than at most $5^{\circ}$ from the correct ones, while for ML and EF the models overall show the correct variation with zodiacal longitude but with values of the synodic arc that may be off by up to $10^{\circ}$ for $\mathrm{EF}$ and up to $20^{\circ}$ for ML. This is confirmed by the data in Table 3 where I have listed in the first four lines the maximum and minimum values and the standard deviations of the differences in position $(\delta \lambda)$ and in time $(\delta t)$ between system $\mathrm{A}_{3}$ ephemerides for the first and last appearances of Venus and observations during the Seleucid Era. ${ }^{13}$

13 For each ephemeris, the initial conditions (planetary position and calendar date) were chosen such that the difference between the model values and the observed values averaged over all 165 observations during the Seleucid era (315-50 BC) was zero. The magnitude of the standard deviation can then be used as a criterion for the quality of fit of the Babylonian models. 

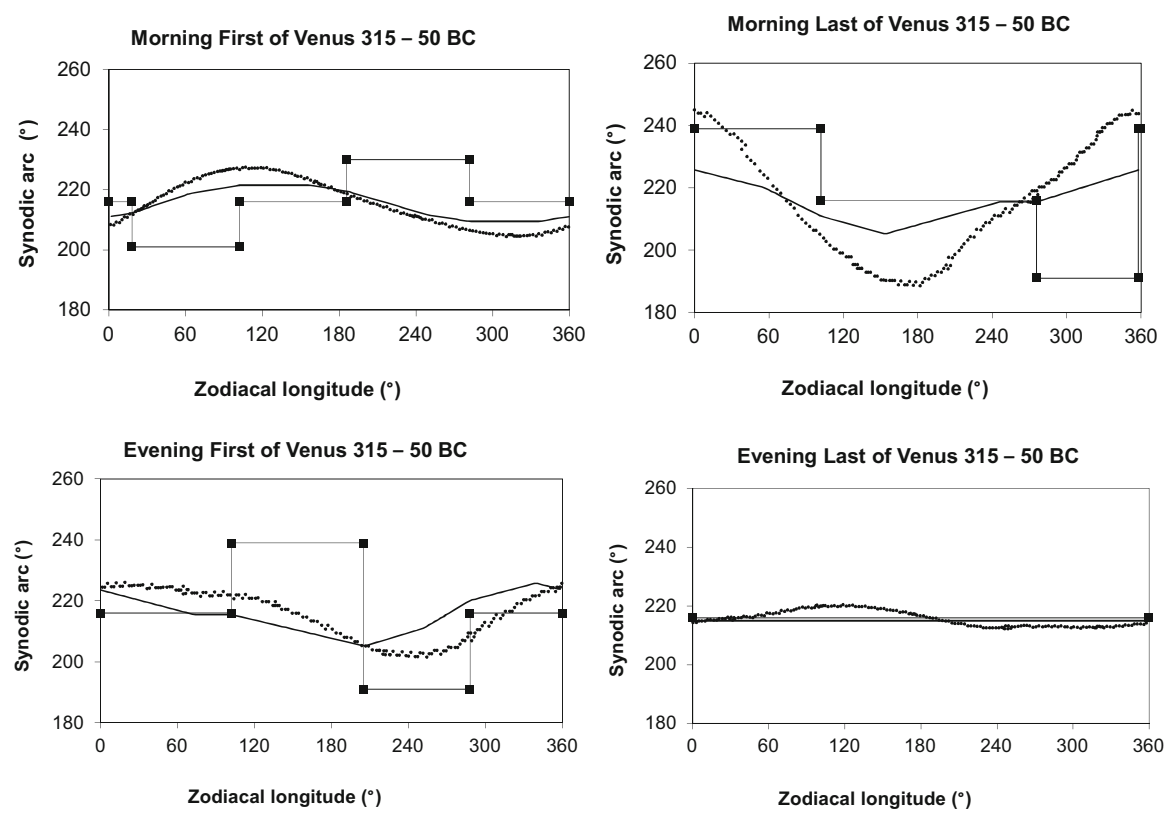

Fig. 4 Synodic arcs for the first and last appearances in the morning and in the evening of Venus as a function of Babylonian zodiacal longitude derived from synthetic observations of Venus from 315 to $50 \mathrm{BC}$ (black dots). Also shown are the Babylonian system $\mathrm{A}_{3}$ model step functions (thin line marked by squares) and the interpolated model values (thick line)

Table 3 System $\mathrm{A}_{3}$ ephemeris of Venus compared to observations 315-50 BC

\begin{tabular}{|c|c|c|c|c|}
\hline \multirow[t]{2}{*}{ Synodic event } & \multicolumn{2}{|c|}{$\delta \lambda\left(^{\circ}\right)$} & \multicolumn{2}{|c|}{$\delta \mathrm{t}$ (days) } \\
\hline & $\max / \min$ & stdev & $\max / \min$ & stdev \\
\hline Morning First & $3.4 /-5.2$ & \pm 2.2 & $6 /-5$ & \pm 3.3 \\
\hline Morning Last & $14.1 /-8.4$ & \pm 6.8 & $11 /-6$ & \pm 5.3 \\
\hline Evening First & $7.8 /-6.3$ & \pm 3.7 & $6 /-5$ & \pm 2.7 \\
\hline Evening Last & $3.9 /-3.5$ & \pm 1.7 & $11 /-8$ & \pm 5.9 \\
\hline Morning Station & $2.3 /-5.4$ & \pm 1.3 & $3 /-3$ & \pm 1.2 \\
\hline Evening Station & $2.6 /-2.7$ & \pm 1.2 & $2 /-2$ & \pm 1.2 \\
\hline (i) & (ii) & (iii) & (iv) & (v) \\
\hline
\end{tabular}

Dates are computed using the Babylonian method in which synodic time intervals (in days) are obtained by adding 17;40 (see column (vi) of Table 2) to the values of the synodic arcs (in degrees). These data show that the system $\mathrm{A}_{3}$ model of Venus provides acceptable fits to the observations of $\mathrm{EL}$ and MF with standard deviations of about $2^{\circ}$ and 6 and 3 days, and rather poor fits for ML and $\mathrm{EF}$ with standard deviations of about $7^{\circ}$ and $4^{\circ}$ and 5 and 3 days, respectively. This accuracy is inferior to that of the system A models of Jupiter and Saturn where planetary longitudes are predicted with an accuracy of about $1^{\circ}$ and dates with an accuracy of a few days (see paper I, Table 4). 

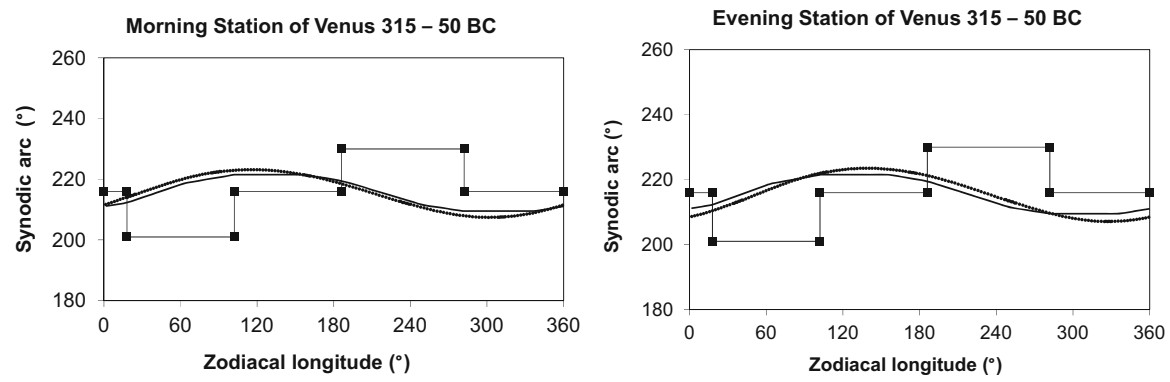

Fig. 5 Same as Fig. 4 but now for the morning and evening stations of Venus. Also shown are the Babylonian system $\mathrm{A}_{3}$ model step function (thin line marked by squares) and the interpolated model values (thick line) for the first appearance of Venus in the morning (MF)

Although the system $\mathrm{A}_{3}$ model of Venus does not include the stations, one might still wonder whether any of its four models would also provide an acceptable fit for the stations. An unexpected positive answer to this question is illustrated in Fig. 5 where the observed variation of the synodic arcs at the stations is compared to the MF system $\mathrm{A}_{3}$ model. The data displayed in Fig. 5 show that the interpolated MF $\mathrm{A}_{3}$ model (full lines) provides an almost perfect fit to the observations (dots). In addition, a closer look at the two panels in Fig. 5 shows that an even better fit would have been obtained if the model was constructed to reproduce the observations of the two stations combined. ${ }^{14}$ The quality of fit of the MF system $\mathrm{A}_{3}$ model to the station data is illustrated in the last two lines of Table 3 where I have listed the maximum and minimum values and the standard deviations of the differences in position and in time between MF system $\mathrm{A}_{3}$ ephemerides for the stations of Venus and station observations during the Seleucid Era. The accuracy of the fit is superior to that of the first and last appearances in Table 3 with standard deviations of about $1^{\circ}$ and 2 to 3 days, comparable in accuracy to the system A ephemerides of Jupiter and Saturn (see paper I, Table 4).

Based on the data in Fig. 5, I would like to turn the question in the previous paragraph around to arrive at the following conclusion: the Babylonian astronomers apparently constructed a system A-type model to fit the variation of the synodic arcs of Venus at its stations and used this model to compute the MF ephemeris preserved on BM 32599. A hopeful aspect of this conclusion is that observations of Venus near its stations are the only way to obtain data needed for the construction of a system A model for Venus but, at the same time, it raises a number of awkward questions: (1) Where are the observations of Venus at its stations, (2) why was this model used to compute longitudes for MF, and (3) how were the models for the other three synodic phases constructed? I will attempt to provide answers to these questions below.

\footnotetext{
14 Notice that the shift to smaller longitudes from morning station to evening station, discussed above in note 11, shows up clearly when one compares the position of the data points with respect to the model curves in the two panels of Fig. 5.
} 


\section{The construction of the system $A_{3}$ model of Venus}

As discussed in Sect. 1, the observation of the stations of Venus was apparently not part of the standard observing program of the Babylonian astronomers as we know it from the Astronomical Diaries. Nevertheless, the fact that the MF system $\mathrm{A}_{3}$ model of Venus provides an almost perfect fit to the variation of the synodic arcs of Venus at its stations strongly suggests that measurements of the position of Venus at its stations relative to nearby Normal Stars must have been available for its construction. Those observations could have been made by some individual astronomer for his own use but never became part of the tradition of what was regularly observed and recorded in Diaries. ${ }^{15}$ So, while it is unlikely that a large body of observations of Venus at its stations was available, it is quite possible that an individual astronomer could have observed them for a couple of decades for his own purposes, which might include developing a model for Venus, especially if he knew already that this is how the models for the outer planets were constructed. ${ }^{16}$

Now let us suppose that this applies to the astronomer who constructed the system $\mathrm{A}_{3}$ model of Venus and who computed the longitude table on BM 32599. Then, taking into account that the text covers the years $403-170 \mathrm{BC}^{17}$ and that the tablet was probably written around $170 \mathrm{BC}$, he might have collected Venus observations from 203 to 178 BC. ${ }^{18}$

To investigate whether 25 years of Venus observations would have been sufficient for the construction of a system A-type model, I have selected observations of the synodic phases of Venus for the period 203-178 BC from the synthetic observational database for the Seleucid era used earlier for the construction of Figs. 2, 3, 4, 5. The synodic arcs derived from the longitudes of Venus at its evening and morning stations during this period are shown in Fig. 6 together with the MF system $\mathrm{A}_{3}$ step function and the interpolated model curve. Data points are represented by open circles with sizes of about $2^{\circ}$, comparable to the accuracy with which synodic arcs can be determined from measurements of positions of Venus with respect to nearby Normal Stars. The clustering of the data points in Fig. 6 is due to the limited timespan of the observing period combined with the backward shift of about $2.5^{\circ}$ in the longitude of Venus after each 8-year period so that after 25 years there are two sets of five clusters of data about $72^{\circ}$ apart, one for evening stations and one for morning stations, each cluster containing three data points in a small longitude range of about $5^{\circ}{ }^{19}$ From the data displayed in Fig. 6, we may conclude that 25 years of observations is indeed sufficient

\footnotetext{
15 I owe this interesting suggestion to John Steele.

16 The central role played by observations of the stations in the construction of the system A models for the outer planets is discussed in paper I.

17 Britton (2001) suggests that the computed longitudes of Venus on the tablet cover the years 419-188 BC. In the next section, I will present the reasons why I prefer the years 403-170 BC.

18 In real life, the observer(s), the scholar and the scribe may have been different persons.

19 For each station, there are five clusters of data points because in 8 years Venus experiences five morning and five evening stations. Since Venus roughly returns to the same position in the zodiac, after 8 years the five clusters of data points are about $72^{\circ}$ apart. Thus, there are two sets of five clusters of data points in Fig. 6, one for each station, which are about $16^{\circ}$ apart, the retrograde arc of Venus from its evening station to its morning station.
} 


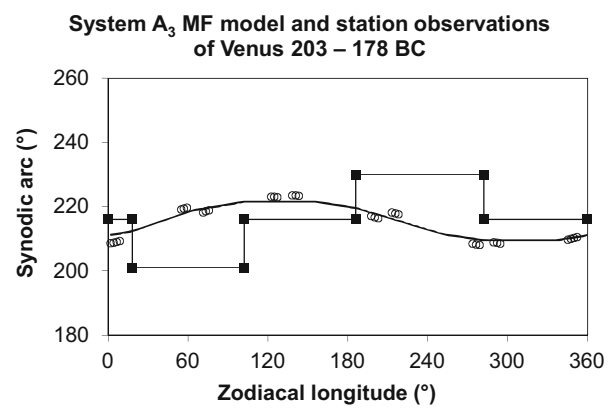

Fig. 6 Synodic arcs for the morning and evening stations of Venus as a function of Babylonian zodiacal longitude derived from observations of Venus between 203 and 178 BC (open circles). Also shown are the Babylonian system $\mathrm{A}_{3}$ model step function (thin line marked by squares) and the interpolated model values (thick line) for the first appearance of Venus in the morning (MF)

for the construction of a system A-type model for the stations of Venus that fits the data. $^{20}$

The analysis above provides an answer to the question about the source of the observations of Venus at its stations and it illustrates the way in which these observations could have been used for the construction of a system A-type model for the stations. However, the question how the system $\mathrm{A}_{3}$ models for the first and last appearances of Venus were constructed remains unanswered. I propose that the author of the text BM 32599 also could have used the observations of Venus from 203-178 BC to compute positions of Venus at its first and last appearances from the observed positions at the stations, using: (1) the dates at which Venus experienced its synodic phenomena and (2) the velocities of Venus in different parts of its orbit. From the dates, he computed time intervals, and multiplying with velocities he computed longitude intervals that were added to or subtracted from the position of Venus at one of its stations.

As discussed in Sect. 1, we know of two theoretical texts, BM 33552 dating from around $320 \mathrm{BC}$ and $\mathrm{BM} 37151$ dating from about $120 \mathrm{BC}$, which contain rather sophisticated models of the motion of Venus. In Table 4, I show the velocity model of the older of these two texts. The actual velocity model of BM 33552 is somewhat more refined than shown in Table 4 because the intervals MS to ML and EF to ES are each split up in two pieces with different and varying velocities. Notice that the model assumes that Venus stands still for 12 days at each stationary point before reversing its direction of motion. This velocity model was available around $170 \mathrm{BC}$ when system $\mathrm{A}_{3}$ was conceived.

In Table 5, I present the same data as in Table 4 but now extracted from the 203-178 BC synthetic observational database. The data in Table 5 show that the longitude and time intervals, going from one synodic phase of Venus to the next one, vary with standard deviations up to $8^{\circ}$ and 8 days. These variations reflect the variable velocity of Venus in the sky due to the combined orbital eccentricities of Venus of $0.7 \%$ and of the Earth of $1.7 \%$. The data in Table 5 also show that the variation in the velocities

\footnotetext{
20 In practice, the author of system $\mathrm{A}_{3}$ may well have used average values of the synodic arcs in each cluster so that he was fitting a curve defined by a fourfold step function through ten data points.
} 
of Venus is much smaller than in the synodic intervals from which the velocities are computed because the synodic arcs and the synodic time intervals vary in phase over large sections of the orbit of Venus. During the relatively short time interval of about 42 days between evening station and morning station, the velocity of Venus experiences variations up to $25 \%$, but the average velocity of $-0.38^{\circ}$ per day over the whole interval is again quite stable.

There is reasonable overall agreement between the observed data in Table 5 and the model in Table 4. The discrepancies in the time intervals are probably related to the observational difficulty to determine the exact date of stationarity when Venus reverses its motion from prograde to retrograde, and vice versa. The discrepancies in the longitude intervals may be related to the fact that the position of Venus cannot be determined by observations with respect to nearby Normal Stars when Venus is near its first and last appearances. The Babylonian values of the velocity of Venus in the different sections of its orbit are correct to within 5\%.

Babylonian astronomers could determine the longitude of Venus by measuring its position with respect to nearby Normal Stars over most of its orbit. But going from evening station to morning station and during periods of about one month before its last appearance in the morning and after its first appearance in the evening the sky is too bright for Normal Stars to be visible so that in those cases the positions of Venus were probably determined by forward or backward extrapolation in time from previous known positions.

The essence of my proposal is that this method of forward and backward extrapolation in time can equally well be used to compute positions of Venus at its first and last appearances using known positions of Venus determined from Normal Star passages or at its stations. Here, I will illustrate this method by using the positions of Venus at its stations, because we know that they must have been available to the author of system $A_{3}$, and by using the velocities of Venus shown in columns (iii) and (v) of Table 4. Differences between the Babylonian values in Table 4 and the observed ones in Table 5 are small so that we can use the Babylonian values of the velocity of Venus of $1.13^{\circ}$ per day from MS to ES and $-0.36^{\circ}$ per day from ES to MS. Once positions of Venus are computed for all first and last appearances between 203 and 178 BC from the time intervals to the last previous station and the average velocities, synodic longitude intervals between successive first and last appearances of the same kind can be derived. The results are graphically presented in Fig. 7 (open circles) together with the system $\mathrm{A}_{3}$ model step functions (thin lines) and the interpolated model values (full drawn lines). ${ }^{21}$ The fact that we encounter in all panels of Fig. 7 a similar clustering of data points as in Fig. 6 is to be expected once we realize that the data in both figures are derived from observed longitudes of Venus at one of its stations over a limited time interval of about 25 years.

By comparing the constructed data in Fig. 7 with the synthetic observational data in Fig. 4, I have verified that the method employed to construct the data is quite successful since in all four panels of Fig. 7 the clusters of data points coincide almost exactly with the synthetic observed data in Fig. 4. From the upper left-hand panel of Fig. 7

\footnotetext{
21 I want to emphasize that Babylonian astronomers did not use graphical representations but, after computing synodic time intervals from dates and synodic arcs from time intervals and velocities, probably wrote and studied the results as parallel columns of numbers.
} 


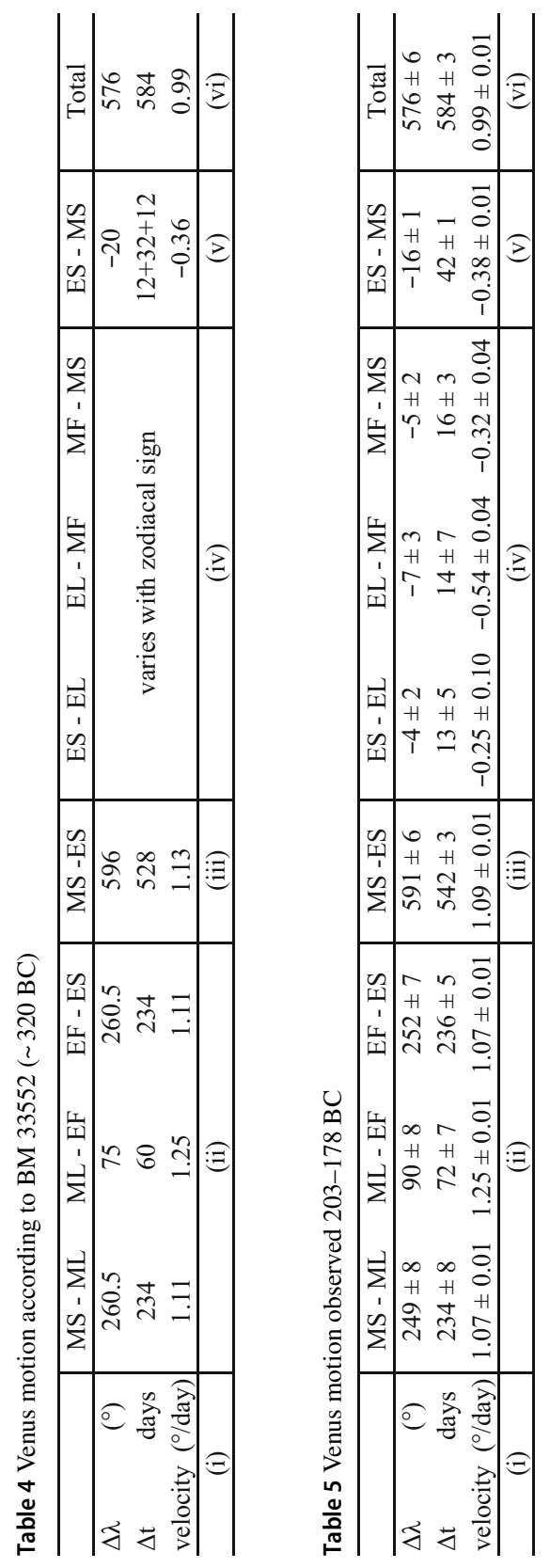



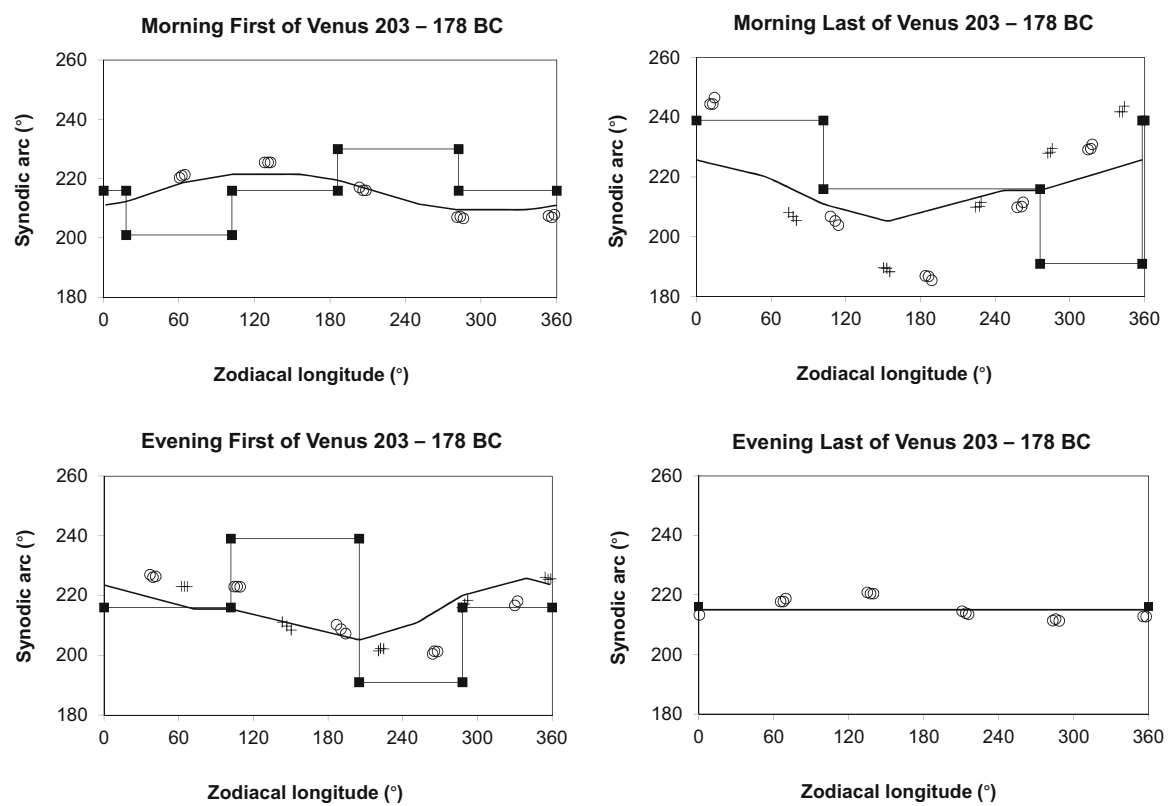

Fig. 7 Synodic arcs for the first and last appearances of Venus as a function of Babylonian zodiacal longitude constructed from observations of Venus between 203 and $178 \mathrm{BC}$ for two different values of the velocity of Venus (open circles and plusses). Also shown are the Babylonian system $\mathrm{A}_{3}$ model step functions (thin lines marked by squares) and the interpolated model values (full drawn lines)

(compare also Fig. 4), it is easy to understand why the Babylonian author of system $\mathrm{A}_{3}$ chose the station model to represent the MF data. It nicely fits the overall variation with longitude, and the extreme values differ only a few degrees with the data points. In fact, if the accuracy of the data construction method would have been less than assumed, here the station model might have provided a close to perfect fit to the MF data. It is also clear that the straight line model to fit the EL data points in the lower right-hand panel of Fig. 7 is a quite adequate approximation to the constructed data points.

It is clear from the upper right-hand and the lower left-hand panels of Fig. 7 that the Babylonian models for the synodic arcs at the last appearance in the morning and the first appearance in the evening of Venus provide rather crude fits to the constructed data points (open circles). The quality of fit significantly improves if we assume that the Babylonian author of system $\mathrm{A}_{3}$ used the average velocity of Venus over its whole orbit of $0.99^{\circ}$ per day (over 584 days) instead of the velocity from morning station to evening station of $1.13^{\circ}$ per day (over 528 days) resulting in the constructed data points represented by crosses. ${ }^{22}$ This would have been an understandable mistake in view of the fact that the constructed positions of Venus at its morning last (ML) and its evening first (EF) involve large time intervals of about 230 and 320 days, about one half of the synodic period of Venus.

\footnotetext{
22 See columns (iii) and (vi) of Tables 4, 5 for these numbers.
} 
With this erroneous velocity, the model for the first appearance of Venus in the evening (EF) fits the constructed data points (crosses) in the lower left-hand panel of Fig. 7 with an accuracy comparable to that of the models for the last appearance in the evening (EL) and for the first appearance in the morning (MF) in Fig. 7. The poor quality of fit of the model for the last appearance in the morning (ML) in the upper right-hand panel of Fig. 7 may be related to the fact that the author of model $\mathrm{A}_{3}$ forced the ML model to have the same parameters as the EF model but differently distributed in the zodiac (see columns (viii)-(x) of Table 2). The amplitudes and the lengths of the three steps in the two models are identical; the first step begins at $12^{\circ}$ Cancer in both models, but the order of the steps is reversed. So it seems that the author of model $\mathrm{A}_{3}$ may have been guided by symmetry considerations to construct a model for EF that is a permutation of the ML model. In view of the symmetrical position of morning last and evening first with respect to the outer conjunction of Venus and the Sun, this approach may be understood.

I conclude that around $170 \mathrm{BC}$ a Babylonian scholar used the method proposed here, or some variant of it, to compute synodic arcs for the first and last appearances of Venus from observational data that he or some colleague had obtained in the previous twenty odd years. Based on these data, he was able to construct the system $\mathrm{A}_{3}$ model used for the computation of the table of longitudes preserved on BM 32599.

\section{The computation of tablet BM 32599}

BM 32599 contains nine columns of sixteen lines on the obverse side and continues with thirteen lines on the reverse side. These are the nine last columns of a tablet that originally contained twenty columns of twenty-nine lines each. Contrary to Babylonian planetary ephemerides, the text only gives longitudes but no dates.

According to the reconstruction of Hamilton and Aaboe (1998) and Britton (2001), the tablet, of which BM 32599 is the remainder, originally listed all first and last appearances of Venus in $29 \times 8=232$ years. Each line gives the longitudes of the $4 \times$ $5=20$ consecutive first and last appearances in one 8-year period of Venus so that the tablet must be read line by line from left to right. The tablet would have started with $s u(\mathrm{EL})$ in the first column and ended with igi (EF) in the last column.

The final reconstruction of the original tablet is shown in Fig. 1 of Britton (2001). For reasons of economy of space, the reconstructed text will not be shown here so that the reader is advised to have a copy of this figure at hand during the discussion below. Columns ([x]ii) to (xx) of the original text are preserved on tablet BM 32599. Zodiacal signs are indicated in the text by numbers $(1=$ Aries, $2=$ Taurus, etc. $)$ and only listed in the first line and whenever a new zodiacal sign is reached while going down in a column. Discrepancies between computed longitudes and those in the text of tablet BM 32599 are underlined. The longitudes in the first seven entries of the two but last column of BM 32599 (column (xviii) in Fig. 1 of Britton 2001) are too large by $0 ; 06^{\circ}$, and the bottom entry of the last column (column (xx) in Fig. 1 of Britton 2001) should have read 7;30 ${ }^{\circ}$ Cancer instead of 8;30 Cancer. Hamilton and Aaboe $(1998,218)$ note that there is no need to compute and write out the full table of 144 lines to cover all synodic phases occurring in one ephemeris period of 1151 years 
because the tablet, of which BM 32599 is the remainder, fully samples the ecliptic for all first and last appearances of Venus with a raster of about $2 ; 30^{\circ}$, sufficiently dense for comparison with observations and for predictions of future events.

Based on a comparison with modern computations, Britton (2001) argues that the tabulated longitudes in the reconstructed tablet represent the first and last appearances of Venus from 419 to $188 \mathrm{BC} \pm 8$ years and that the tablet was written around 186 $\mathrm{BC} \pm 8$ years. Since it is somewhat unusual for a mathematical astronomical text to contain past phenomena rather than future predictions, Britton (2001) also considers the possibility that the tablet contains predictions for the period $184 \mathrm{BC}$ to 47 $\mathrm{AD} \pm 8$ years but he ends up preferring the early dating.

Aaboe and Hamilton (1998) and Britton (2001) have suggested that going down in the table the longitudes in each line were computed by subtracting a small shift $w^{\prime}$ (i) (see column (xii) of Table 2) from the longitudes in the previous line. That the entries in the table may in fact have been computed in this way is supported by the colophon at the bottom of the reverse side of the tablet where the procedure for computing kur (MF) is partly preserved. Tabulating and computing the first and last appearances of Venus in this way is numerically very convenient and elegant because the shift remains constant over many steps, while only occasionally a zone boundary is transgressed and-if so-always only one per step. Filling the tablet line by line from left to right by computing sequences of positions of consecutive similar synodic phases (EL to EL to EL..., MF to MF to MF..., etc.) is numerically much more complicated because the synodic arcs w(i) are large $\left(190^{\circ}-240^{\circ}\right.$, see column (x) of Table 2$)$, and in most cases more than one zone boundary is transgressed per step.

So it seems plausible that the tablet, of which BM 32599 is the remainder, was filled line by line starting with a sequence of twenty positions of Venus at consecutive first and last appearances during one 8-year period. Once these twenty initial positions were computed by the usual algorithms of the system A computational scheme (using the parameters listed in columns (viii)-(x) of Table 2), the further line-by-line filling of the table by using the small increments w'(i) in column (xi) of Table 2 was computationally an easy and straightforward task. This line-by-line filling could have been done downward, as suggested by the colophon on the tablet, starting with initial values in line 1 but might just as well have been carried out upward starting with initial values at the bottom of the table in line 29 . The latter method is computationally even a little simpler because small increments are added rather than subtracted from line to line and the interpolation algorithm over zone boundaries is the usual one of system A. In fact, the mistakes made by the scribe in writing tablet BM 32599 suggest that the latter approach was followed because the computational error in the seventh entry of kur (MF) in the one but last column of the tablet (column (xviii) of Fig. 1 of Britton 2001)) where a zone boundary is transgressed, propagates upward in the tablet all the way to line one. It seems difficult to explain this series of errors otherwise. The other mistake in tablet BM 32599 in the bottom entry of igi (EF) in the last column of the tablet looks like an isolated scribal error.

Now that we understand the way in which the tablet was computed, the one question still to be answered is the choice of the initial conditions of the computation. We know from previous studies that the Babylonian astronomers chose integer values of the dates and integer or simple fractional sexagesimal values of the longitudes as 
initial conditions for the computation of their planetary ephemerides. ${ }^{23}$ We have also seen that the author of BM 32599 probably started out by computing the positions of twenty consecutive first and last appearances during one 8-year period using the system $\mathrm{A}_{3}$ step function parameters in columns (viii) to (x) of Table 2 and then used those positions for a further line-by-line filling of the tablet. Thus, it seems obvious to look for a sequence of four "nice" sexagesimal numbers in the four leftmost columns of the reconstructed tablet as initial conditions. Those are found in the last ten lines of the reconstructed tablet in Fig. 1 of Britton (2001). While the longitudes in any one of those ten lines could have been used as starting points for the further line-by-line filling of the tablet, upward and downward, it is most natural to start from the bottom line and fill the tablet line by line upward so that the most probable initial conditions of the calculation are the longitudes of the four successive synodic phases of Venus in the lower left-hand corner of the tabulation: $26 ; 00^{\circ}$ Pisces at the last appearance in the evening $(s u), 24 ; 30^{\circ}$ Pisces at the first appearance in the morning $(k u r), 12 ; 00^{\circ}$ Sagittarius at the last appearance in the morning $(D U)$ and $15 ; 30^{\circ}$ Aquarius at the first appearance in the evening ( $i g i)$. The fact that these initial conditions are located in the lower left-hand corner of the table may be interpreted as another independent argument in support of my suggestion that the tablet was filled from the bottom line upward rather than from the top line downward.

Is it possible to identify the observations of Venus on which the choice of these initial conditions is based? In an attempt to do so, I note that during the 25 years of observations on which the development of system $\mathrm{A}_{3}$ of Venus may have been based Venus reached its evening station on 18 February 179 BC while very close to the star $\eta$ Piscium, the Normal Star near the beginning of the Babylonian fixed zodiac. On this day, the distance between Venus and $\eta$ Piscium was $0.4^{\circ}$ in longitude and $1.5^{\circ}$ in latitude. This is quite a special observation because during the previous one hundred years there were only four observations where Venus at one its stations had been as close or closer to one of the Normal Stars.

To investigate whether this observation may have played a role in the selection of the initial conditions for the computation of BM 32599, I list in the first four columns of Table 6 the position of $\eta$ Psc and the dates and longitudes of Venus at its two stations and its first and last appearances in 179/178 BC, starting with its evening station on 18 February $179 \mathrm{BC}$. These data are taken from the synthetic database. Ecliptic longitudes of Venus are converted to Babylonian longitudes by adding $5.5^{\circ} \cdot{ }^{24}$ Of these six synodic phenomena, only the dates could be directly observed and the longitude of Venus at its evening station. No stars are visible at the first and last appearances of Venus, and none of the Normal Stars were nearby when Venus reached its morning station. However, the same method used earlier to convert time intervals to longitude intervals by using the velocity of Venus could have been applied here to compute the longitudes of Venus by starting out from the observation of Venus at its evening station $0.4^{\circ}$ to the West of the Normal Star $\eta$ Psc. ${ }^{25}$ This is illustrated in columns (v)-(x) of Table 6 where the

\footnotetext{
23 See for instance paper I, section 8 .

24 See note 8.

25 Since the longitudes of Normal Stars in Babylonian star catalogues are given in units of whole degrees (Sachs 1952), I have started the calculation in Table 6 by adopting $2^{\circ}$ for the longitude of $\eta$ Psc. In the star
} 
Table 6 Initial conditions for the computation of BM 32559

\begin{tabular}{|c|c|c|c|c|c|c|c|c|c|}
\hline \multicolumn{4}{|c|}{ Synthetic observations $179 / 178 \mathrm{BC}$} & \multicolumn{5}{|c|}{ Babylonian observations 179/178 BC } & \multirow{2}{*}{$\begin{array}{c}\text { Tablet } \\
\lambda \text { (bab) } \\
\left({ }^{\circ}\right)\end{array}$} \\
\hline Obs & Date & $\begin{array}{c}\lambda(\mathrm{ecl}) \\
\left({ }^{\circ}\right)\end{array}$ & $\begin{array}{c}\lambda(\mathrm{bab}) \\
\left(^{\circ}\right)\end{array}$ & Date & $\begin{array}{c}\Delta \mathrm{T} \\
\text { (days) }\end{array}$ & $\begin{array}{c}\text { Vel } \\
(\% / \text { day })\end{array}$ & $\begin{array}{l}\Delta \lambda \\
\left({ }^{\circ}\right)\end{array}$ & $\begin{array}{c}\lambda(\mathrm{bab}) \\
\left({ }^{\circ}\right)\end{array}$ & \\
\hline$\eta$ Psc & & 356.6 & 2.1 & & & & & 2.0 & \\
\hline \multirow[t]{2}{*}{ Venus ES } & 18 -Feb & 356.1 & 1.6 & $23-\mathrm{Feb}$ & & & & 1.6 & \\
\hline & & & & & 15 & -0.36 & -5.4 & & \\
\hline \multirow[t]{2}{*}{ Venus EL } & 7-Mar & 350.6 & 356.1 & 10-Mar & & & & 356.2 & 356.0 \\
\hline & & & & & 5 & -0.36 & -1.8 & & \\
\hline \multirow[t]{2}{*}{ Venus MF } & 13-Mar & 347.1 & 352.6 & 15-Mar & & & & 354.4 & 354.5 \\
\hline & & & & & 19 & -0.36 & -6.8 & & \\
\hline \multirow[t]{2}{*}{ Venus MS } & 2-Apr & 341.8 & 347.3 & 3-Apr & & & & 347.6 & \\
\hline & & & & & 234 & 1.13 & 264.4 & & \\
\hline \multirow[t]{2}{*}{ Venus ML } & $27-\mathrm{Nov}$ & 234.6 & 240.1 & $23-\mathrm{Nov}$ & & & & 252.0 & 252.0 \\
\hline & & & & & 56 & 1.13 & 63.3 & & \\
\hline Venus EF & 30-Jan & 315.5 & 321.0 & 18-Jan & & & & 315.3 & 315.5 \\
\hline (i) & (ii) & (iii) & (iv) & (v) & (vi) & (vii) & (viii) & (ix) & (x) \\
\hline
\end{tabular}

dates in column (v) are the actually observed dates. The corresponding time intervals are given in column (vi) of Table 6, average velocities from the Babylonian velocity model in Table 4 are given in column (vii), computed longitude intervals are given in column (viii), and resulting Babylonian longitudes of Venus are given in column (ix). The dates in column (vi) are chosen in such a way that the resulting longitudes in column (ix) are as close as possible to the longitudes of the initial conditions of the computation in BM 32599 listed in column (x) of Table 6.

The credibility of the method proposed here to select the initial conditions for the computation of BM 32599 depends on the credibility of the adopted dates in column (v) of Table 6. If those dates deviate more than can be explained by observational errors or by atmospheric fluctuations from the ones listed in column (ii), which are computed for a nominal atmosphere in Babylon characterized by an atmospheric extinction of 0.27 magnitudes per air mass, the method fails. Since it is difficult to observationally determine the exact date of standstill of Venus, an observer may be easily off by \pm 5 days in establishing the dates of the stations. In my study of Babylonian observations of Venus (de Jong 2012, 397), I have shown that variations in the atmospheric extinction (weather) may cause variations in the dates of up to \pm 3 days for $E L$ and $M F$ and of up to \pm 15 days for ML and EF. So it seems justified to conclude that the method proposed here, or some variant of it, may have been used by the Babylonian astronomer who composed BM 32599 to start his computation with initial values based on the observation of Venus at its evening station very close to the Normal Star $\eta$ Psc one hour after sunset in Babylon on the 12th day of the month Aiaru in year 132 of the Seleucid Era (23 February 179 BC).

Footnote 25 continued

catalogue in section 8 of BM 36609+, a longitude of 3;20 is listed for $\eta$ Psc, but this is probably $1^{\circ}$ to $2^{\circ}$ too large due to a systematic error in that section of the catalogue (Roughton et al. 2004, 553-554). 
Based on this choice of initial conditions, the tablet, of which BM 32599 is the remainder, covers the years $403 \mathrm{BC}$ to $170 \mathrm{BC}$ and it may have been composed in Babylon around $1705 \mathrm{BC}$. It was probably computed with the purpose of creating a raster of longitudes with mazes of about $2.5^{\circ}$ that could be used to predict future positions of Venus at its first or last appearances if a previous position was available. Dates could then also have been predicted by using the relation $\overline{\Delta t}=\overline{\Delta \lambda}+17 ; 40$.

Acknowledgements I would like to thank Peter Huber, Alexander Jones, Mathieu Ossendrijver and John Steele for a number of interesting suggestions and advice.

\section{Compliance with ethical standards}

Conflict of interest The author states that there is no conflict of interest.

Open Access This article is distributed under the terms of the Creative Commons Attribution 4.0 International License (http://creativecommons.org/licenses/by/4.0/), which permits unrestricted use, distribution, and reproduction in any medium, provided you give appropriate credit to the original author(s) and the source, provide a link to the Creative Commons license, and indicate if changes were made.

\section{References}

Aaboe, A. and Huber, P.J. 1977. A text concerning subdivision of the synodic motion of Venus from Babylon: BM 37151. In Ancient Near Eastern Studies in Memory of J.J. Finkelstein, Connecticut, 1-4.

Aaboe, A., Britton, J.P., Henderson, J.A., Neugebauer, O., and Sachs, A.J. 1991. Saros cycle dates and related Babylonian astronomical texts.Transactions of the American Philosophical Society, 81(6), $1-71$.

Brack-Bernsen, L. and Hunger, H. 2005/2006. On the Atypical Astronomical Cuneiform Text E. Archiv für Orientforschung 51:96-107.

Britton, J.P. 2001. Remarks on a system a text for Venus: ACT 1050. Archive for History of Exact Sciences 55: 525-554.

Britton, J.P. 2002. Treatments of annual phenomena in Cuneiform sources. In Under one sky: Astronomy and mathematics in the ancient Near East, ed. J.M. Steele and A. Imhausen, 21-78., Alter Orient und Altes Testament 297 Münster: Ugarit-Verlag.

Britton, J.P., and C.B.F. Walker. 1991. A 4th Century Babylonian model for Venus: BM 33552. Centaurus 34: $97-118$.

Hamilton, N.T., and A. Aaboe. 1998. A Babylonian Venus text computed according to system A: ACT No. 1050. Archive for History of Exact Sciences 53: 215-221.

Huber, P. 1958. Über den Nullpunkt der babylonischen Ekliptik. Centaurus 5: 192-208.

Hunger, H., Sachs, A.J., and Steele, J.M. 2001. Astronomical diaries and related texts from Babylonia, Vol. V: Lunar and Planetary Texts, Vienna (ADRT V).

de Jong, T. 2012. Babylonian observations of Venus: Arcus visionis, atmospheric extinction and observational practice. Journal for the History of Astronomy 43: 391-409.

de Jong, T. 2016. Babylonian Astronomy 1880-1950: The Players and the Field. In A mathematician's journeys: Otto Neugebauer and modern transformations of ancient science, ed. A. Jones, Chr. Proust and J.M. Steele, 265-302. New York: Springer.

de Jong, T. 2017. On the origin of the lunar and solar periods in Babylonian lunar theory. In Studies on the ancient exact sciences in honour of Lis Brack-Bernsen, ed. J.M. Steele and M. Ossendrijver, 105-126. Berlin.

de Jong, T. 2019. A study of Babylonian planetary theory I. The outer planets. Archive for History of Exact Sciences 73: 1-37.

Neugebauer, O. 1955. Astronomical cuneiform texts, vol. 3. London: ACT.

Neugebauer, O. 1975. A history of ancient mathematical astronomy, vol. 3. Berlin: HAMA. 
Neugebauer, O., and A.J. Sachs. 1967. Some atypical astronomical cuneiform texts. Journal of Cuneiform Studies 21: 183-217.

Ossendrijver, M. 2012. Babylonian mathematical astronomy. Berlin: Procedure Texts.

Reiner, E. and Pingree, D. 1975. The Venus Tablet of Ammisaduqa, Bibliotheca Mesopotamica vol. II, part 1, Undena Publications, Malibu.

Roughton, N.A., J.M. Steele, and C.B.F. Walker. 2004. A late Babylonian normal and Ziqpu star text. Archive for History of Exact Sciences 58: 537-572.

Sachs, A.J. 1952. A late Babylonian star catalogue. Journal of Cuneiform Studies 6: 146-150.

Sachs, A.J. and Hunger, H. 1988, 1989, 1996. Astronomical diaries and related texts from Babylonia, Vols. I, II and III, Vienna (ADRT I-III).

Publisher's Note Springer Nature remains neutral with regard to jurisdictional claims in published maps and institutional affiliations. 